\title{
BMJ Open Sequelae of multidrug-resistant tuberculosis: protocol for a systematic review and meta-analysis
}

\author{
Kefyalew Addis Alene, ${ }^{1,2}$ Archie C A Clements, ${ }^{1}$ Emma S McBryde, ${ }^{3}$ \\ Ernesto Jaramillo, ${ }^{4}$ Knut Lonnroth, ${ }^{5}$ Debebe Shaweno, ${ }^{6}$ Kerri Viney ${ }^{1,5}$
}

To cite: Alene KA, Clements ACA, McBryde ES, et al. Sequelae of multidrugresistant tuberculosis: protocol for a systematic review and meta-analysis. BMJ Open 2018;8:e019593. doi:10.1136/ bmjopen-2017-019593

- Prepublication history and additional material for this paper are available online. To view these files, please visit the journal online (http://dx.doi. org/10.1136/bmjopen-2017019593).

Received 13 September 2017 Revised 14 November 2017 Accepted 17 November 2017

Check for updates

${ }^{1}$ Research School of Population Health, College of Health and Medicine, Australian National University, Canberra, Australia ${ }^{2}$ Institute of Public Health, College of Medicine and Health Sciences, University of Gondar, Gondar, Ethiopia

${ }^{3}$ Australian Institute of Tropical Health and Medicine, James Cook University, Townsville, Queensland, Australia ${ }^{4}$ Global TB Programme, World Health Organization, Geneva, Switzerland

${ }^{5}$ Department of Public Health Sciences, Karolinska Institutet, Stockholm, Sweden

${ }^{6}$ Department of Medicine, University of Melbourne, Melbourne, Victoria, Australia

Correspondence to Kefyalew Addis Alene; kefyalew.alene@anu.edu.au

\section{ABSTRACT}

Introduction The sequelae of multidrug-resistant tuberculosis (MDR-TB) are poorly understood and inconsistently reported. We will aim to assess the existing evidence for the clinical, psychological, social and economic sequelae of MDR-TB and to assess the healthrelated quality of life in patients with MDR-TB.

Methods and analysis We will perform a systematic review and meta-analysis of published studies reporting sequelae of MDR-TB. We will search PubMed, SCOPUS, ProQuest, Web of Science and PsychINF0 databases up to 5 September 2017. MDR-TB sequelae will include any clinical, psychological, social and economic effects as well as health-related quality of life that occur after MDR-TB treatment or illness. Two researchers will screen the titles and abstracts of all citations identified in our search, extract data, and assess the scientific quality using standardised formats. Providing there is appropriate comparability in the studies, we will use a random-effects meta-analysis model to produce pooled estimates of MDRTB sequelae from the included studies. We will stratify the analyses based on treatment regimen, comorbidities (such as HIV status and diabetes mellitus), previous TB treatment history and study setting.

Ethics and dissemination As this study will be based on published data, ethical approval is not required. The final report will be disseminated through publication in a peerreviewed scientific journal and will also be presented at relevant conferences.

PROSPERO registration number CRD42017073182.

\section{INTRODUCTION}

Multidrug-resistant tuberculosis (MDR-TB) is a global threat that has several documented sequelae in affected patients. ${ }^{1}$ MDR-TB is caused by Mycobacterium tuberculosis and is defined as TB that is resistant to at least isoniazid and rifampicin, the two most effective first-line, anti-TB drugs. ${ }^{2}$ Approximately half a million people are estimated to have MDR-TB annually. ${ }^{3}$ In 2016, 490000 new MDR-TB and 110000 rifampicin-resistant tuberculosis (RR-TB) cases were estimated to have occurred. ${ }^{3}$ In the same year, 240000 people were estimated to have died due to MDR-TB or RR-TB worldwide. ${ }^{3}$ The treatment of

\section{Strengths and limitations of this study}

- This will be the first published systematic review and meta-analysis that comprehensively assesses multidrug-resistant tuberculosis (MDR-TB) sequelae in people who were diagnosed with or treated for MDR-TB.

- The search will be conducted without geographical, language and time restrictions.

- The search will be performed with the help of a specialised librarian experienced in medical literature searching.

- The data reporting will adhere to the Preferred Reporting Items for Systematic reviews and MetaAnalyses Protocols.

- A potential limitation of this study will be a large degree of heterogeneity between published studies.

MDR-TB is more costly, toxic and has a longer duration than the treatment of drug-susceptible TB. ${ }^{45}$ As a result, the drugs used to treat MDR-TB, as well as the disease itself, can lead to a range of side effects and consequences, ranging from mild, self-limiting to life-threatening conditions. ${ }^{6-8}$ However, the burden of MDR-TB sequelae has not been quantifiedessential information for understanding the global burden of disease and associated social and economic costs.

Sequelae refer to conditions that are the consequence of a previous disease or injury. ${ }^{9}$ Post-treatment sequelae are contextually defined in our study as any consequence of MDR-TB treatment that occurred after a full course of treatment or after exposure to TB medicine, even if the exposure was not a full course. Post-illness sequelae include the long-term and short-term consequences of MDR-TB. ${ }^{10-12}$ The term 'MDR-TB sequelae' here refers to both sequelae arising from illness and sequelae arising as side effects of treatment. This includes any clinical, psychological, social and economic sequelae as well as health-related quality of life that occurs after MDR-TB treatment or illness. 
A variety of clinical sequelae occur in treated or untreated MDR-TB patients due to drug side effects or due to the disease itself. The clinical sequelae related to the disease comprise a range of long-lasting complications such as impaired pulmonary function, chronic obstructive pulmonary disease (COPD), lung scarring (fibrosis), bronchiectasis that leads to recurrent infections, TB (including acquired drug resistance) and heart failure. $^{1314}$ The common post-treatment sequelae that occur as a result of acute or ongoing drug side effects include hearing loss, visual disturbances, hepatitis, renal dysfunction, bone marrow suppression and peripheral neuropathy. ${ }^{15}$ There are also other side effects that are specific to the drugs used to treat MDR-TB. ${ }^{16} 17$

The economic sequelae of MDR-TB are a result of both direct medical costs incurred by diagnosis and treatment and indirect costs including productivity loss, sale of assets and interests on loans during treatment, due to ill health or long absences from the workplace. ${ }^{18}$ While there are also costs to the health service, we will focus only on financial sequelae imposed on an individual due to MDR-TB treatment or illness.

The psychological and social effects of MDR-TB are diverse. Depression, anxiety, substance use, stigma, discrimination and distress are thought to be the common psychological and social issues reported among patients with MDR-TB. ${ }^{12}{ }^{19}$ In addition, like any other chronic illness, MDR-TB can also affect the health-related quality of life. ${ }^{20}$

It has been reported in previous studies that MDR-TB sequelae increase the risk of TB recurrence ${ }^{21}$ and death. ${ }^{22}$ The magnitude and severity of MDR sequelae vary, and the risk factors for these sequelae are diverse. Some of the risk factors for MDR-TB sequelae are underlying comorbidities such as HIV infection and diabetes mellitus (DM), previous history of $\mathrm{TB}$ treatment, the pattern of drug resistance and the treatment regimen used. ${ }^{22-24}$

Serious MDR-TB sequelae further increase the population impact of the disease. ${ }^{25}$ The MDR-TB surveillance systems in several countries have reported only MDR-TB cases and their treatment outcomes. However, MDR-TB sequelae also need to be monitored and quantified to estimate the true burden of the disease, evaluate the potential benefit of preventive methods and measure effectiveness of treatment regimens. Post-MDR-TB sequelae may persist even after successful treatment outcomes. ${ }^{25}$ Further, a variety of sequelae and complications can occur in both treated and untreated cases of MDR-TB. ${ }^{26}$

Despite the fact that several individual studies have reported on the most common MDR-TB sequelae and their risk factors, there is no published systematic review and meta-analysis that shows pooled estimates of MDR-TB sequelae. Having a comprehensive understanding of MDR-TB sequelae and identifying the main risk factors for MDR-TB are important for clinicians, policy-makers, programme managers, health professionals and researchers in order to better understand how MDR-TB affects patients over the longer term. It may inform more effective long-term follow-up and medical care of these patients and prevention of the sequelae. Therefore, the aim of this systematic review and meta-analysis is to review the existing literature and quantify the burden of MDR-TB sequelae and to identify risk factors for these sequelae.

\section{OBJECTIVES}

The objectives of our study are:

1. to review the clinical sequelae and health-related quality of life in patients with MDR-TB treatment or illness

2. to determine the prevalence of psychological and social sequelae in patients with MDR-TB

3. to measure the economic burden of MDR-TB faced by patients and affected families

4. to identify risk factors for MDR-TB sequelae.

\section{METHODS}

\section{Protocol}

We have developed this systematic review and meta-analysis protocol according to the Preferred Reporting Items for Systematic Reviews and Meta-Analyses Protocols (online supplementary appendix 1). ${ }^{27}$ The review protocol for the study has been registered in the International Prospective Register of Systematic Reviews (CRD42017073182).

\section{Search strategy}

We will search for studies in PubMed, SCOPUS, ProQuest, Web of Science and PsychINFO databases up to 5 September 2017, with no limitations on year or language of publication. We will restrict our search to human studies (only). We will develop search terms in accordance with the Medical Subject Headings (MeSH) thesaurus using a combination of keywords. For MDR-TB, we will use keywords "Tuberculosis, Multidrug-Resistant"[Mesh], "drug-resistan*”, "multidrug resistan*”, "mdr*", "xdr*", "tuberculosis" and "tb"; for clinical, psychological, social and economic sequelae as well as quality of life, we will use keywords that are related to each term. The full electronic search strategies are included in the supplementary information (online supplementary appendix 2). We have developed our search strategy with the help of a medical librarian skilled in doing systematic reviewers. In addition, we will perform a hand search of the reference lists of retrieved articles and abstracts from the International Union Against Tuberculosis and Lung Disease Journal (2004-2017), the European Respiratory Journal, Tuberculosis and Lancet Respiratory Medicine, for additional papers. We will contact authors of relevant papers by email when additional information is required.

\section{Selection of studies}

All citations identified through our search strategy will be imported into EndNote V.X7 (Thompson Reuters, New York, New York, USA). After excluding duplicated studies from our EndNote Library, the title and abstracts of the 
remaining articles will be independently assessed by two reviewers (KAA and DS), with final inclusion of studies to be decided through consensus. Differences will be resolved by discussion with a third author $(\mathrm{KV})$. If the title and abstract of a paper appear relevant, the full text of the paper will be reviewed. We will include studies based on the following eligibility criteria.

\section{Participants}

Studies examining clinical, psychological, social and economic sequelae as well as health-related quality of life in persons with MDR-TB treatment or illness.

\section{Outcomes}

Studies explicitly reporting on clinical, psychological, social and economic sequelae, as well as health-related quality of life

- Clinical sequelae: pulmonary function, COPD, bronchiectasis, fibrosis, aspergilloma, granuloma/calcification, heart failure, hearing impairment, visual disturbance, liver toxicity, renal dysfunction, bone marrow suppression and peripheral neuropathy. It will also include any disability that occurred as a result of MDR-TB or its treatment.

- Health-related quality of life: physical, psychological, social, mental and environmental domains of quality of life.

- Social and psychological sequelae: depression, anxiety, psychosis, stigma, discrimination, distress and mental health disorders including substance abuse.

- Economic sequelae: cost of illness, productivity loss, sales of assets, interest on loans and loss of job.

\section{Study designs}

Observational studies, such as retrospective cohort, prospective cohort and cross-sectional studies, as well as interventional studies will be included.

We will exclude studies that focus only on drug-susceptible TB.

\section{Data extraction}

Data will be extracted from eligible studies by two independent reviewers (KAA and DS), and information will be collated in a Microsoft Excel 2016 spreadsheet (Microsoft, Redmond, Washington, USA). The data extraction spreadsheet will be piloted on five different handsearched papers. We will collect data on the outcomes of our study from each individual paper.

We will record post-MDR-TB sequelae as prevalence, cumulative incidence, incidence density or incidence proportion as reported in the individual papers. We will also record relative risks or odds ratios to calculate relative risk compared with controls if data are available.

We will also extract the following data from each paper: name of first author, year of publication, country in which the study was conducted, study design, number of included study participants (that will be used as a denominator for all of the outcomes), participation rate, baseline socioeconomic indicators, demographic factors (including mean age in years and percentage of male patients), diagnostic method to ascertain MDR-TB, mean number of drugs to which the patient's isolate was resistant, percentage of patients with HIV infection and percentage of patients with DM at baseline (if known). We will also collect data on the mean number of drugs provided to MDR-TB patients, the mean duration of MDR-TB treatment in months, treatment regimen (ie, standardised vs individualised and ambulatory or community-based vs hospitalised), the percentage of patients who received a fluoroquinolone antibiotic (levofloxacin, moxifloxacin, gatifloxacin or sparfloxacin) as part of their treatment regimen, the percentage of patients who received second-line injectable drugs and the percentage of patients who received cycloserine.

\section{Quality assessment}

Two authors (KAA and DS) will assess the methodological quality of the included studies, including the risk of bias in the selection of the study groups (ie, people diagnosed with MDR-TB) and outcome ascertainment using the Newcastle-Ottawa Scale. ${ }^{28}$

\section{STATISTICAL ANALYSIS}

\section{Data analysis}

Data extracted from selected studies will be presented in evidence tables. We will undertake the descriptive statistical analyses of the extracted data using STATA V.14.1. Because of anticipated heterogeneity, we plan to use a Bayesian random-effects meta-analysis model to obtain pooled estimates of prevalence across studies for the outcomes of interest. This model will assume that there is heterogeneity of true-effect sizes (ie, MDR-TB sequelae), and our goal will be to estimate the mean of this value. ${ }^{29}$ The method will also allow direct interpretation of credible intervals of the posterior-effect estimates and investigations of sources of heterogeneity by considering underlying risk factors. ${ }^{29}{ }^{30}$ The posterior parameters will be estimated using a large number of vague but proper prior distributions and data information using a Bayesian Markov Chain Monte Carlo simulation approach with Gibbs sampling, employed by WinBUGS V.1.4.3 software (Medical Research Council Biostatistics Unit, Cambridge, UK). After an initial burn-in and observation of convergence, assessed using Brooks-Gelman criteria, ${ }^{31}$ the model will be run subsequently for 100000 iterations to provide values for the posterior distributions. Posterior distributions will be summarised according to mean and 95\% credible interval limits.

\section{Assessment of heterogeneity and reporting biases}

The magnitude of variation or heterogeneity between studies will be quantitatively measured by index of heterogeneity $\left(\mathrm{I}^{2}\right.$ and its $\left.\mathrm{CI}\right) .{ }^{32} \mathrm{I}^{2}$ values of $25 \%, 50 \%$ and $75 \%$ are assumed to represent low, medium and high heterogeneity, respectively. The significance of the heterogeneity will be determined by $\chi^{2}$ for $Q$ statistics at a $\mathrm{P}$ value less than 0.05 . Funnel plots will be used to 
detect potential publication bias and small-study effects. A P value $<0.05$ will be considered as indicative of statistically significant publication bias. For outcomes with more than 10 individual studies, we will use the Egger method to assess asymmetry.

\section{Subgroup and sensitivity analysis}

We will conduct a subgroup analysis to assess the effects of each study characteristic on the primary outcomes of the study. Stratified analysis will be performed by: the study setting (ie, high and low TB burden countries, based on the WHO high burden county lists for TB), ${ }^{33}$ treatment regimens (standardised and individualised treatment), HIV prevalence $(0$ and $>0 \%)$ and history of previous TB treatment $(<75 \%$ and $>75 \%)$. Sensitivity analyses will be also performed to verify the robustness of the study conclusion and to assess the impacts of methodological quality, sample size and analysis methods on the results.

\section{DISCUSSION}

The number of MDR-TB cases and associated postMDR-TB sequelae has increased in several countries and has posed additional challenges for national TB prevention and control programs. ${ }^{5} 131425$ Expanding healthcare provided by national $\mathrm{TB}$ programmes to address the consequences or sequelae of MDR-TB is essential for effective TB management. Although previous studies have shown that in many settings the clinical, psychological and financial burden of MDR-TB is significantly higher than for person with drug-susceptible TB, ${ }^{6-8} 18$ the sequelae of MDR-TB are poorly understood and inconsistently reported.

This systematic review and meta-analysis will provide pooled estimates of MDR-TB sequelae among people who were diagnosed with or treated for MDR-TB. It will also identify the main risk factors for MDR-TB sequelae. Identifying the main risk factors for long-term and short-term sequelae of MDR-TB using a comprehensive systematic review and meta-analysis will provide empirical evidence necessary for clinicians, health professionals, researchers and decision-makers to better understand the risk factors, policy implications, future research needs and programming priorities for the treatment, care and follow-up of persons with MDR-TB.

Contributors KAA, KV and EJ conceived the study. KAA developed the search strategy and drafted the protocol. KV improved the drafted protocol. KL and EJ provided expertise to the section on psychosocial and economic burden of MDR-TB. ACAC, ESM, DS and KAA critically revised the manuscript for methodological and intellectual content. All authors have read and approved the final manuscript.

Funding This research received no specific grant from any funding agency in the public, commercial or not-for-profit sectors.

Competing interests None declared.

Patient consent Not required.

Provenance and peer review Not commissioned; externally peer reviewed.

Author note If there is a need to amend this protocol, the date of each amendment and the reason for the change will be described.
Open Access This is an Open Access article distributed in accordance with the Creative Commons Attribution Non Commercial (CC BY-NC 4.0) license, which permits others to distribute, remix, adapt, build upon this work non-commercially, and license their derivative works on different terms, provided the original work is properly cited and the use is non-commercial. See: http://creativecommons.org/ licenses/by-nc/4.0/

(c) Article author(s) (or their employer(s) unless otherwise stated in the text of the article) 2018. All rights reserved. No commercial use is permitted unless otherwise expressly granted.

\section{REFERENCES}

1. Marais BJ. The global tuberculosis situation and the inexorable rise of drug-resistant disease. Adv Drug Deliv Rev 2016;102:3-9.

2. Millard J, Ugarte-Gil C, Moore DA. Multidrug resistant tuberculosis. BMJ 2015;350:h882.

3. World Health Organization. Global tuberculosis report 2017. Geneva, Switzerland: World Health Organization, 2017.

4. World Health Organization. Companion handbook to the WHO guidelines for the programmatic management of drug-resistant tuberculosis. Geneva, Switzerland: World Health Organization, 2014.

5. van den Hof S, Collins D, Hafidz F, et al. The socioeconomic impact of multidrug resistant tuberculosis on patients: results from Ethiopia, Indonesia and Kazakhstan. BMC Infect Dis 2016;16:470.

6. Törün T, Güngör G, Ozmen I, et al. Side effects associated with the treatment of multidrug-resistant tuberculosis. Int J Tuberc Lung Dis 2005;9:1373-7.

7. Baghaei P, Tabarsi P, Dorriz D, et al. Adverse effects of multidrugresistant tuberculosis treatment with a standardized regimen: a report from Iran. Am J Ther 2011;18:e29-34.

8. Geerligs WA, Van Altena R, De Lange W, et al. Multidrug-resistant tuberculosis: long-term treatment outcome in the Netherlands. Int $\mathrm{J}$ Tuberc Lung Dis 2000;4:758-64.

9. Oxford English Dictionary. "art n.1". Oxford: Oxford University Press.

10. Leimane V, Riekstina V, Holtz TH, et al. Clinical outcome of individualised treatment of multidrug-resistant tuberculosis in Latvia: a retrospective cohort study. Lancet 2005;365:318-26.

11. Dheda K, Gumbo T, Gandhi NR, et al. Global control of tuberculosis: from extensively drug-resistant to untreatable tuberculosis. Lancet Respir Med 2014;2:321-38.

12. Bassili A, Fitzpatrick C, Qadeer E, et al. A systematic review of the effectiveness of hospital- and ambulatory-based management of multidrug-resistant tuberculosis. Am J Trop Med Hyg 2013;89:271-80.

13. Byrne AL, Marais BJ, Mitnick CD, et al. Chronic airflow obstruction after successful treatment of multidrug-resistant tuberculosis. ERJ Open Res 2017;3:26.

14. Chakaya J, Kirenga B, Getahun H. Long term complications after completion of pulmonary tuberculosis treatment: A quest for a public health approach. J Clin Tuberc Other Mycobact Dis 2016;3:10-12.

15. Tahaoğlu K, Törün T, Sevim T, et al. The treatment of multidrugresistant tuberculosis in Turkey. N Engl J Med 2001;345:170-4.

16. Iseman MD. Treatment of multidrug-resistant tuberculosis. $N$ Engl J Med 1993;329:784-91.

17. Yang TW, Park HO, Jang HN, et al. Side effects associated with the treatment of multidrug-resistant tuberculosis at a tuberculosis referral hospital in South Korea: a retrospective study. Medicine 2017;96:e7482.

18. Tanimura $\mathrm{T}$, Jaramillo $\mathrm{E}$, Weil $\mathrm{D}$, et al. Financial burden for tuberculosis patients in low- and middle-income countries: a systematic review. Eur Respir J 2014;43:1763-75.

19. Acha J, Sweetland A, Guerra D, et al. Psychosocial support groups for patients with multidrug-resistant tuberculosis: five years of experience. Glob Public Health 2007;2:404-17.

20. Sharma R, Yadav R, Sharma M, et al. Quality of life of multi drug resistant tuberculosis patients: a study of north India. Acta Med Iran 2014;52:448-53.

21. Chen MY, Lo YC, Chen WC, et al. Recurrence after successful treatment of multidrug-resistant tuberculosis in Taiwan. PLoS One 2017; 12:e0170980.

22. Pietersen E, Ignatius E, Streicher EM, et al. Long-term outcomes of patients with extensively drug-resistant tuberculosis in South Africa: a cohort study. Lancet 2014;383:1230-9.

23. Chiang CY, Enarson DA, Yu MC, et al. Outcome of pulmonary multidrug-resistant tuberculosis: a 6-yr follow-up study. Eur Respir J 2006;28:980-5.

24. Gelmanova IY, Ahmad Khan F, Becerra MC, et al. Low rates of recurrence after successful treatment of multidrug- 
resistant tuberculosis in Tomsk, Russia. Int $J$ Tuberc Lung Dis 2015;19:399-405.

25. Singla N, Singla R, Fernandes S, et al. Post treatment sequelae of multi-drug resistant tuberculosis patients. Indian J Tuberc 2009;56:206-12.

26. Menon B, Nima G, Dogra V, et al. Evaluation of the radiological sequelae after treatment completion in new cases of pulmonary, pleural, and mediastinal tuberculosis. Lung India 2015;32:241-5.

27. Shamseer L, Moher D, Clarke M, et al. Preferred reporting items for systematic review and meta-analysis protocols (PRISMA-P) 2015: elaboration and explanation. BMJ 2015;349:97647.

28. Wells G, Shea B, O'Connell D, et al. Newcastle-Ottawa quality assessment scale cohort studies, 2014.
29. Smith TC, Spiegelhalter DJ, Thomas A. Bayesian approaches to random-effects meta-analysis: a comparative study. Stat Med 1995;14:2685-99.

30. Warn DE, Thompson SG, Spiegelhalter DJ. Bayesian random effects meta-analysis of trials with binary outcomes: methods for the absolute risk difference and relative risk scales. Stat Med 2002;21:1601-23.

31. Brooks SP, Gelman A. General methods for monitoring convergence of iterative simulations. J Comput Graph Stat 1998;7:434-55.

32. Higgins JP, Thompson SG. Quantifying heterogeneity in a metaanalysis. Stat Med 2002;21:1539-58.

33. WHO. Use of high burden country lists for tuberculosis by WHO in the post-2015 era. Geneva, Switzerland: WHO, 2015. 